\title{
Migraine headache associated with multiple sclerosis: Results from the Nationwide Inpatient Sample (NIS)
}

\author{
Sura S. Khuder ${ }^{a}$ Sreekiran Thotakura (iD) ${ }^{b}$ Fouzia Siddiqui (iD ${ }^{b}$ Nabeel A. Herial (iD ${ }^{b}$ Sadik A. Khuder (iD ${ }^{c}$ and Boyd \\ M. Koffman (iD) $b$
}

Corresponding author(s): boyd.koffman@utoledo.edu

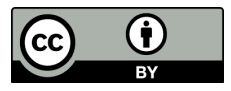

\footnotetext{
${ }^{a}$ College of Medicine and Life Sciences, University of Toledo Toledo, OH 43614, USA, ${ }^{b}$ Department of Neurology, University of Toledo, Toledo, OH 43606, USA, and ${ }^{c}$ Department of Medicine, University of Toledo, Toledo, $\mathrm{OH} 43614, \mathrm{USA}$.
}

Background: Multiple Sclerosis (MS) is a dysimmune process that targets axons in the central nervous system. A high prevalence of migraine headache has been reported among MS patients. Objecctives: In this study, we investigated headache prevalence and association in MS patients. Methods: The Health Cost and Utilization Project (HCUP) Nationwide Inpatient Sample (NIS) data was utilized. MS hospitalizations ("cases") were identified by ICD-9-CM code 340 . Non-MS Hospitalizations ("controls") were matched to cases $1: 1$ by age and gender. Results: We identified 18955 MS patients between 2010 and 2013. The prevalence of migraine among MS patients $(7 \%)$ was significantly higher than the control group (2.8\%). Non-specified Migraine constituted the highest percentages of migraine subtype in both groups. All the subtypes of headache, except migraine without aura, were significantly higher among MS patients compared to the control group. Among MS patients, young age, obesity, depression, chronic lung disease, white race, and female gender were significant predictors of migraine. Conclusions: Our study provides prevalence data for different subtypes of headache and supports the association of young age, obesity, depression, chronic lung disease, white race, and female gender as risk factors for migraine.

Multiple Sclerosis | autoimmune diseases | comorbidities | HCUP data

M

ultiple sclerosis (MS) is a chronic inflammatory process that targets myelinated axons in the central nervous system. MS is an immune-mediated disease in which activated immune cells invade the central nervous system and cause inflammation characterized by myelin loss, varying degrees of axonal pathology, and progressive neurological dysfunction. The clinical features of MS encompass an extremely wide range of neurological symptoms (1).
The etiology of MS is not yet completely understood (2). A complex interplay of genetic and environmental associated factors is currently considered for the etiology of the disease $(3,4)$.

People with multiple sclerosis (MS) have an increased incidence of headaches, although the comorbidity of headaches and MS is poorly understood (5). Several studies suggest a high prevalence of headache among MS patients and with migraine representing the most common type of primary headache. The association between migraine and MS was observed in two case-control series $(6,7)$. A population-based study from Sicily reported a doubling of headache prevalence in MS patients compared to controls (8). Only one study did not detect a statistically significant difference in headaches between MS patients and historical controls (9), possibly due to very high prevalence of primary headaches in the local population. In this study, we investigated the prevalence and risk for migraine among patients with MS in the United States utilizing a nationwide database.

\section{Material and Methods}

Data used in this study is from the Healthcare Cost and Utilization Project (HCUP) sponsored by the Agency for Healthcare Research and Quality (AHRQ). HCUP combines data from state

\section{Submitted: 02/19/2020, published: 04/25/2021.}

Freely available online through the UTJMS open access option 
organizations, hospital associations, private data organizations, and the Federal government to create a national information resource consisting of patient-level health care data. The largest collection of longitudinal hospital care data in the United States is included in HCUP beginning in 1988. The HCUP database represents $96 \%$ of the U.S. population and includes over 32 million observations. HCUP consists of multiple databases, however the database used in this study is the Nationwide Inpatient Sample (NIS) for the years 2010-2013. This database has inpatient data from over 1,000 hospitals and up to 44 states in the United States.

Subjects included in the study consisted of individuals who were at least 18 years of age and had an ICD-9-CM diagnosis code (340) for MS. The NIS database allows up to 25 diagnoses per patient. Since MS is an immune-mediated process, controls were excluded if they had one or more of over 100 different autoimmune disease ICD-9 diagnosis codes or were under the age of 18. MS patients and controls were included and matched by age ( \pm 5 years) and sex. One control was matched to each MS patient (18955 MS and 18955 Control).

The comorbid conditions used in this analysis totaled 29 and were variables included in the NIS database obtained from the AHRQ comorbidity software. This comorbidity software assigns variables that identify comorbidities in discharge records using ICD-9-CM codes. The 29 comorbidities included in this study are listed in Table 4.

All statistical analyses were conducted using SAS version 9.2 (SAS Institute, Cary, NC) and R. Frequency distributions between categorical variables were assessed using the $\chi^{2}$ test. Logistic regression models were utilized to compare patients with and without MS for 29 different comorbidities. The survey logistic procedures in $\mathrm{R}$ were used in this analysis to include the weight variable provided in the database. We evaluated risk factors associated with headache such as age, gender, race, smoking, alcohol, obesity, depression, and comorbidities. Regarding race, other races ("other") served as the reference to which Whites, Blacks, and Hispanics were compared.

\section{Results}

Between 2010 and 2013, there were 18955 admissions with MS listed as principal diagnosis for admission. Comparisons of MS patients and controls on demographic variables revealed equal distributions for age, gender, and region (Table 1). Compared to controls, there were significantly less admissions for Hispanic and lother" among the MS group, and significantly increased admissions among Blacks for the MS group.

The prevalence of migraine among MS patients (7\%) was significantly higher than the control group $(2.8 \%)$. All the subtypes of migraine were significantly higher among MS patients compared to the control group (Table 2). Migraine unspecified constituted the highest percentages of migraine in both groups. Migraine with and without aura and unspecified were significantly higher among MS patients compared to the control group.

Predictors of migraine headache among MS patients are presented in Table 3. The likelihood of migraine decreased as a function of age and is increased in females. Compared to the category of "other", Whites were more likely to suffer from migraine. Comorbidities significantly increased the risk of migraine among MS patients.

Table 4 presents the distribution comorbidities between migraine and nonmigraine MS patients. Comorbidities that increase the risk of migraine include chronic lung, depression, and obesity. Comorbidities that decrease the risk of migraine include congestive heart failure (CHF), hypertension, paralysis, and alcohol.

Table 1. Hospital admissions with migraine among MS and Controls for 2010-2013.

\begin{tabular}{cccc}
\hline \hline Variable & $\begin{array}{c}\text { MS } \\
\text { N=18955 }\end{array}$ & $\begin{array}{c}\text { Control } \\
\text { N=18955 }\end{array}$ & p-value \\
\hline Age & & & \\
(mean \pm SD) & $45.1 \pm 13.5$ & $45.0 \pm 14.0$ & 0.898 \\
Gender & & & \\
Female \% & 73.1 & 73.1 & 1 \\
Race & & & \\
Race White\% & 61.9 & 61.7 & $<0.001$ \\
Black\% & 25.1 & 18.8 & \\
Hispanic\% & 9.2 & 12.6 & \\
Other\% & 3.8 & 6.9 & \\
Region & & & \\
Northeast & 24.2 & 23.8 & 0.748 \\
Midwest & 25.6 & 25.6 & \\
South & 34.0 & 34.5 & \\
West & 16.2 & 16.1 & \\
Comorbidities \% & 45.6 & 46.6 & 0.051 \\
Smoking\% & 20.3 & 17.9 & $<0.001$ \\
& & & \\
\hline
\end{tabular}

Table 2. Distribution of headache between MS and Controls.

\begin{tabular}{cccccc}
\hline \hline Headache & MS & & Controls & & p-value \\
& $\mathrm{N}$ & $\%$ & $\mathrm{~N}$ & $\%$ & \\
\hline Migraine & & & & & \\
With aura & 36 & 0.2 & 15 & 0.1 & 0.0032 \\
Without aura & 44 & 0.2 & 16 & 0.1 & $<0.0001$ \\
Hemiplegic, & & & & & \\
Menstrual, & & & & & \\
variant forms & 48 & 0.3 & 33 & 0.2 & 0.2247 \\
Unspecified & 1196 & 6.3 & 462 & 2.4 & $<0.0001$ \\
Total & 1321 & 7.0 & 524 & 2.8 & $<0.0001$ \\
\hline
\end{tabular}


Table 3. Predictors of Migraine headache among MS Patients.

\begin{tabular}{ccccc}
\hline \hline Coefficient & OR & $\begin{array}{c}\mathbf{9 5 \%} \text { CI } \\
\text { Lower }\end{array}$ & Upper & p-value \\
& & & & \\
\hline Age* & & 3.12 & 5.00 & $<2 \mathrm{e}-16$ \\
$18-39$ & 3.95 & 2.26 & 3.53 & $<2 \mathrm{e}-16$ \\
$40-59$ & 2.82 & 2.88 & 4.21 & $<2 \mathrm{e}-16$ \\
Female gender & 3.48 & & & \\
Race** & & 1.07 & 2.19 & 0.019 \\
$\begin{array}{l}\text { White } \\
\text { Black }\end{array}$ & 1.53 & 0.77 & 1.61 & 0.567 \\
Hispanic & 1.11 & 0.79 & 1.76 & 0.410 \\
Smoking & 0.99 & 0.85 & 1.15 & 0.875 \\
Comorbidities & 1.32 & 1.16 & 1.49 & $<0.0001$ \\
& & & & \\
\hline Reference category (age): $\geq 60$ years $* *$ Reference category $($ race $):$ Other.
\end{tabular}

\section{Discussion}

This study aimed to examine the prevalence of migraine among MS patients based on hospital admissions data. Data suggests that there is a statistically significant association between migraine and MS, with MS patients being more than twice as likely to report migraine as controls.

The prevalence of migraine among MS patient in this study was $7 \%$. This result is similar to those reported in other studies (8-17). The results of the more recent studies based on International Headache Society criteria for diagnosis and classification of headaches showed that the lifetime prevalence of migraine is higher in MS patients (more than 50\%) than in controls and general population, suggesting an increased risk of migraine and a possible association between these two conditions.

Migraine prevalence decreased with age and this is consistent with other studies. As headache prevalence rates decrease with age (18) one could assume that the differences between MS patients with and without migraine are due to the older age of the nonmigraine sufferers.

The higher prevalence of headache in women compared to men has been attributed to the effect of female sex hormones. Epidemiological studies consistently show higher prevalence of migraine in women, with a female to male ratio in the order of 2.3:1 $(18,19)$.

Migraine prevalence was higher in Whites compared to Blacks and this is consistent with those reported in the literature (20). Compared to Asian and other races, the prevalence of migraine was higher in Whites. Epidemiological studies have reported higher prevalence in Caucasian as compared to Asian populations (21).

Among MS patients, individuals with migraine suffered from depression at significantly higher rates than migraine-free counterpart. This finding is consistent with previous literature data. Migraine is reported as comorbid with depression in the general population (22) and also in the MS population (23). Moreover, depression was mentioned as a significant determinant of the severity of comorbid migraine in multiple sclerosis (24).

MS migraineurs reported alcohol intake at significantly lower rates than non-migraineur MS patients. Alcohol is mentioned as one of many factors that can trigger migraine attacks (25). Therefore, alcohol may be avoided not just by those with migraine. One study observed that both those who experience migraine and those who experience headache were likely to have low intake of alcohol (26).

Obesity increased the risk of migraine among MS patients. Obesity was mentioned as a risk factor for migraine in the literature, although migraine by itself was not more prevalent in obese patients. Obesity was also associated with the frequency and severity of migraine (27), but the causal link between obesity and migraine remains to be determined. Another study reported an association between obesity and migraine that did not adjust for frequent intake of acute pain drugs (28).

Our study has several important strengths. We used a national inpatient database representing $96 \%$ of the U.S. population with over 32 million patient records utilized in analysis, enabling a clearer picture of the prevalence of headache among MS patients on a country-wide basis. Furthermore, the headache types identified in this study are based on doctor-diagnosed diseases, and not self- 
Table 4. Distribution of comorbidities between Migraine and Non Migraine MS patients.

\begin{tabular}{|c|c|c|c|}
\hline & Migraine \% & $\begin{array}{c}\text { Non } \\
\text { Migrane \% }\end{array}$ & p-value \\
\hline Alcohol abuse & 0.7 & 1.4 & 0.0380 \\
\hline deficiency anemias & 10.1 & 9 & 0.1877 \\
\hline $\begin{array}{l}\text { Rheumatoid arthritis and } \\
\text { collagen vascular diseases }\end{array}$ & 2.6 & 1.9 & 0.0975 \\
\hline Chronic blood loss anemia & 0.2 & 0.2 & 1 \\
\hline Congestive heart failure (CHF) & 0.5 & 1.3 & 0.0101 \\
\hline Chronic pulmonary disease & 17.8 & 11.0 & $<0.0001$ \\
\hline Coagulopathy & 1.3 & 1.4 & 0.739 \\
\hline Depression & 32.6 & 23.7 & $<0.0001$ \\
\hline Diabetes, uncomplicated & 10.4 & 11.6 & 0.1929 \\
\hline Diabetes with complication & 1.7 & 1.6 & 1 \\
\hline Drug abuse & 5.2 & 4.8 & 0.5671 \\
\hline Hypertension & 29.3 & 32.4 & 0.0202 \\
\hline Hypothyroidism & 9.3 & 9.0 & 0.714 \\
\hline Liver disease & 0.5 & 0.9 & 0.1621 \\
\hline Lymphoma & 0.2 & 0.1 & 1 \\
\hline Fluid and electrolyte disorders & 13.1 & 13.7 & 0.542 \\
\hline Metastatic cancer & 0 & 0.1 & 0.3469 \\
\hline Other neurological disorders & 0.1 & 0.1 & 1 \\
\hline Obesity & 14.2 & 9.9 & $<0.0001$ \\
\hline Paralysis & 9.0 & 10.9 & 0.03638 \\
\hline Peripheral vascular disorders & 0.6 & 0.9 & 0.4195 \\
\hline Psychoses & 9.1 & 7.4 & 0.03018 \\
\hline Pulmonary circulation disorders & 0.2 & 0.4 & 0.3612 \\
\hline Renal Failure & 0.9 & 1.3 & 0.248 \\
\hline Solid tumor without metastasis & 0.2 & 0.3 & 0.8598 \\
\hline Peptic ulcer disease & 0 & 0 & 1 \\
\hline Valvular disease & 2.0 & 1.3 & 0.03985 \\
\hline Weight loss & 1.5 & 2.3 & 0.09363 \\
\hline
\end{tabular}

reporting by patients. In the case of autoimmune diseases, which include rare diseases and diseases with considerable clinical heterogeneity and complex case definitions, the collection of data through self-reporting involves a high probability of reporting bias.

There are also several potential weaknesses in the design of this study. First, the data is limited to hospitalized patients, and therefore not all patients suffering from multiple sclerosis were investigated. However, the HCUP data may not reflect the true prevalence of migraine. Furthermore, the group included in this study was sicker and possibly showing higher prevalence of certain types of migraine. Second, we were unable to adjust for significant covariates such as family history, medication use, and diet. Third, multiple admission for the same patient might be problematic. However, there is no reason to believe that the pattern of admission is different between MS patients and the controls. Moreover, we included only patients admitted with MS as primary diagnosis.

Potential future directions:

1. The inpatient NIS database was used because it utilizes a large population-based dataset, but MS is typically treated on an outpatient basis. One potential extension of work may include finding and exploiting a large population-based outpatient database to more realistically model migraine in MS.

2. Is it possible that chronic CNS inflammation can present with migraine-like symptoms but is actually an MS exacerbation? One could argue that migraine and MS are both more prevalent in females and that migraine at MS presentation is simply a coincidence. However, there is a literature suggesting that the initial presentation of MS may include symptoms consistent with migraine. MS is an immune-mediated disorder, and in one regard could be considered a chronic encephalitis. Clinical recognition of new headache symptoms with features of migraine as an entity worthy of evaluation may lead to earlier diagnosis of MS and potentially better outcomes with earlier treatment. Another potential extension of work may include seeking a database that allows linkage of subjects with medications, diagnoses, durable medical equipment (DME) over time to evaluate time course from diagnosis to use of DME such as cane, walker, or wheelchair over time to evaluate the modification of symptoms with treatment.

\section{Conclusion}

We conducted a large population-based case-control study evaluating the prevalence of migraine in MS cases compared to age- and gender-matched controls. Migraine is common among patients with MS; factors associated with increased likelihood of migraine in MS 
include young age, obesity, depression, chronic lung disease, white race, and female gender. Findings were similar in direction though smaller in magnitude compared to previous studies which primarily included much smaller numbers of MS cases. One immediate implication from this study is that potential risk factors that increase the likelihood of migraine in MS (obesity, depression, and chronic lung disease) should be modified when possible. Primary care providers, psychiatrists, and neurologists are the clinicians in the best position to modify these risk factors.

\section{Conflict of interest}

Authors declare no conflict of interest.

\section{Institutional Review Board approval}

Data sets from the Healthcare Cost and Utilization Project (HCUP) healthcare databases, including the Nationwide Inpatient Survey are deidentified and publicly/commercially available. These

1. Noseworthy JH, Lucchinetti C, Rodriguez M, Weinshenker BG. (2000) Multiple sclerosis. N Engl J Med 343, 938-952.

2. Zettl UK, Stuve O, PatejdI R. (2012) Immune-mediated CNS diseases: a review on nosological classification and clinical features. Autoimmunity reviews 11, 167-173.

3. Comabella M, Khoury SJ. (2012) Immunopathogenesis of multiple sclerosis. Clinical Immunology 142, 2-8.

4. Mix E, Meyer-Rienecker H, Hartung HP, Zettl UK. (2010) Animal models of multiple sclerosis-potentials and limitations. Neurobiol 92, 386-404.

5. Villani $\mathrm{V}$ et al. (2008) Primary headache and multiple sclerosis: preliminary results of a prospective study. Sci 29(Suppl 1), S146-S148.

6. Watkins SM, Espir M. (1969) Migraine and multiple sclerosis. J Neurol Neurosurg Psychiatry 32. 35-37.

7. Rolak LA, Brown S. (1990) Headaches and multiple sclerosis: a clinical study and review of the literature. $J$ Neurol 237, 300-302.

8. Nicoletti $A$ et al. (2008) Headache and multiple sclerosis: a population-based case-control study in Catania, Sicily. Cephalalgia 28, 1163-1169.

9. Putzki N et al. (2009) Prevalence of migraine, tension-type headache and trigeminal neuralgia in multiple sclerosis. Eur $J$ Neurol 16, 262-267.

10. Freedman MS, Gray TA. (1989) Vascular headache: a presenting symptom of multiple sclerosis. Can J Neurol Sci 16, 63-66.

11. Clifford DB, Trotter JL. (1984) Pain in multiple sclerosis. Arch Neurol 41, 12701272.

12. Kister I, Caminero AB, Herbert J, Lipton RB. (2010) Tension-type headache and migraine in multiple sclerosis. Pain Headache Rep 14, 441-448.

13. D'Amico D et al. (2004) Prevalence of primary headaches in people with multiple sclerosis. Cephalalgia 24, 980-984.

14. Gee JR, Chang J, Dublin AB, Vijayan N. (2005) The association of brainstem lesions with migraine-like headache: an imaging study of multiple sclerosis. Headache 45, 670-677. data sets do not involve "human subjects" (as defined by federal regulations and guidance). Therefore, their use requires neither IRB review, an exempt determination, nor are users required to complete the NIH human subjects training.

\section{Authors' contributions}

SSK: data collection, data analysis, manuscript writing; ST: data collection, data analysis, manuscript editing; FS: Study concept and design, manuscript editing; NAH: Study concept and design, manuscript editing; SAK: Study concept and design; data collection, project development, data analysis, manuscript editing and content revision; BMK: Study concept and design, project development, data analysis, manuscript writing; manuscript editing, and content revision.

All authors read and approved the final document.

15. Yetimalar $Y$, Secil $Y$, Inceoglu AK, Eren S, Basoglu M. (2008) Unusual primary manifestations of multiple sclerosis. N Z Med J 121, 47-59.

16. Martinelli Boneschi $F$ et al. (2008) Lifetime and actual prevalence of pain and headache in multiple sclerosis. Mult Scler 14, 514-521.

17. Vacca G et al. (2007) Multiple sclerosis and headache co-morbidity. A casecontrol study. Neurol Sci 28, 133-135.

18. Victor TW, Hu X, Campbell JC, Buse DC, Lipton RB. (2010) Migraine prevalence by age and sex in the United States: a life-span study. Cephalalgia 30, 1065-1072.

19. Stovner LJ et al. (2007) The global burden of headache: A documentation of headache prevalence and disability worldwide. Cephalalgia 27, 193-210.

20. Robbins MS, Lipton RB. (2010) The epidemiology of primary headache disorders. Semin Neurol 30, 107-119.

21. Stewart WF, Lipton RB, Liberman J. (1996) Variation in migraine prevalence by race. Neurology 47, 52-59.

22. Breslau N, Lipton RB, Stewart WF, Schultz LR, Welch KMA. (2003) Comorbidity of migraine and depression: investigating potential etiology and prognosis. Neurology 60, 1308-1312.

23. Kister $I$ et al. (2010) Migraine is comorbid with multiple sclerosis and associated with a more symptomatic MS course. J Headache Pain 11, 417-425.

24. Villani $V$ et al. (2012) Determinants of the severity of comorbid migraine in multiple sclerosis. Neurol Sci 33, 1345-1353.

25. Bartleson JD, Cutrer FM. (2010) Migraine update. Diagnosis and treatment. Minnesota Medicine 93, 36-41.

26. Rist PM, Buring JE, Kurth T. (2015) Dietary patterns according to headache and migraine status: A cross-sectional study. Cephalgia 35, 767-775.

27. Bigal ME, Liberman JN, Lipton RB. (2006) Obesity and migraine: a population study. Neurology 66, 545-550.

28. Yu $S$ et al. (2012) Body mass index and migraine: a survey of the Chinese adult population. J Headache Pain 13, 531-536 